\title{
Dexamethasone protects against arsanilic acid-induced rat vestibular dysfunction through the BDNF and JNK 1/2 signaling pathways
}

\author{
YAN LUO $^{1,2}$, DAOGONG ZHANG ${ }^{1},{\text { YUELING } \mathrm{CHEN}^{1}, \text { ZHONGSHENG CAO }^{2} \text { and ZHAOMIN FAN }}^{1}$ \\ ${ }^{1}$ Department of Otolaryngology-Head and Neck Surgery, Shandong Provincial Hospital Affiliated to Shandong University, \\ Jinan, Shandong 250021; ${ }^{2}$ Department of Otolaryngology, The Second Affiliated Hospital of \\ Soochow University, Suzhou, Jiangsu 215004, P.R. China
}

Received April 8, 2018; Accepted November 12, 2018

DOI: $10.3892 / \mathrm{mmr} .2019 .9835$

\begin{abstract}
The brain-derived neurotrophic factor (BDNF) and c-Jun NH 2-terminal kinase (JNK) signaling pathways are therapeutic targets to prevent degeneration in the central nervous system. Dexamethasone (DXMS), a glucocorticoid, protects against vestibular brain injury, however, the molecular mechanisms have yet to be fully elucidated. To investigate whether the BDNF and JNK signaling pathways are involved in the protective effects of DXMS in rats with vestibular dysfunction, a rat model of severe vestibular deficits was established by middle ear injection of arsanilic acid (AA; $100 \mathrm{mg} / \mathrm{ml} ; 0.05 \mathrm{ml})$. After 3 days, rat symptoms and behavior scores with vestibular disorders were detected. In brain tissues, histopathological alterations, cell apoptosis, expression levels and patterns of BDNF signaling pathway-associated BDNF, tyrosine receptor kinase $\mathrm{B}(\mathrm{TrKB})$ and $\mathrm{K}^{+} / \mathrm{Cl}^{-}$cotransporter isoform 2 (KCC2), and the expression of apoptosis-related cleaved-caspase 3 and the JNK signaling pathway were detected. It was identified that DXMS relieved AA-induced vestibular dysfunction, leading to improvement in rat behavior scores to normal levels, minimizing brain damage at the histopatholojnnkngical level, reducing cell apoptosis, enhancing the expression of BDNF, TrKB and $\mathrm{KCC} 2$, and downregulating cleaved-caspase 3 and phosphorylated-JNK1/2 in brain tissues. Together, these findings indicated the protective effect of DXMS on AA-induced rat vestibular dysfunction, and that activating BDNF and inhibiting JNK singling pathways were the underlying mechanisms. In addition, with additional
\end{abstract}

Correspondence to: Professor Zhaomin Fan, Department of Otolaryngology-Head and Neck Surgery, Shandong Provincial Hospital Affiliated to Shandong University, 324 Jingwuweiqi Road, Jinan, Shandong 250021, P.R. China

E-mail: fanent@126.com

Key words: dexamethasone, arsanilic acid, vestibular dysfunction, brain-derived neurotrophic factor singling pathway, c-Jun $\mathrm{NH}$ 2-terminal kinase singling pathway treatment of mifepristone (RU486), a specific glucocorticoid agonist, all the events elicited by DXMS mentioned above in the AA-treated rat rats were reversed. In conclusion, DXMS was identified as a therapeutic agent targeting the BDNF and JNK singling pathways for AA-induced rat vestibular dysfunction.

\section{Introduction}

The vestibular system is responsible for the perception of spatial location, posture and movement, and its dysfunction or selective impairment leads to vestibular deficits, including motion impairment, postural deviation, disorientation, nystagmus, vertigo and dizziness (1-3), during which apoptotic mediators promote those pathological alterations (4). Vestibular compensation is considered to be behavioral recovery from lesions to the peripheral vestibular system, and is widely used to investigate the plasticity of central vestibular system and recuperation of vestibular functions (5).

Brain-derived neurotrophic factor (BDNF) regulates synaptic transmission and plasticity (6), contributes to neurogenesis (7), and is involved in spatial orientation (8), motor and cognitive skills (9); thus, it has been closely associated with the development of the vestibular system. BDNF, an activity-dependent release substance, functions through binding to its affinity receptor tyrosine receptor kinase $\mathrm{B}$ (TrkB) in the vestibular system (5). BDNF deficiency causes severe loss and degeneration of vestibular neurons, and contributes to vestibular dysfunction $(10,11)$. A previous study demonstrated that the upregulation of BDNF accelerates the process of vestibular compensation (7). Therefore, BDNF appears to be important in vestibular compensation.

In previous years, corticosteroids have been identified to have beneficial effects on symptoms and vestibular functions (12), however, evidence of corticosteroid therapy for vestibular disorders remains insufficient $(13,14)$. Dexamethasone (DXMS), a well-known glucocorticoid, exerts a high therapeutic index for activation of vestibular nucleus neurons, and subsequently accelerates vestibular compensation (15). However, to the best of our knowledge, detailed elucidation of the roles of DXMS and its 
mechanisms in vestibular compensation has been rare. In the present study, an arsanilic acid (AA)-induced rat vestibular dysfunction model was established to investigate whether DXMS is a therapeutic option for this disease. Following DXMS (1 and $3 \mathrm{mg} / \mathrm{kg} /$ day) treatment, vestibular symptoms, behavioral scores and brain damage were assessed, and the expression levels and distributions of BDNF, tyrosine receptor kinase $\mathrm{B}\left(\right.$ TrkB) and $\mathrm{K}^{+} / \mathrm{Cl}^{-}$cotransporter isoform 2 (KCC2), cleaved-caspase 3 and c-Jun NH 2-terminal kinase (JNK)1/2 were detected in brain sections. In addition, RU486, an inhibitor of glucocorticoid receptor, was introduced for intervention of the effects of DXMS. The data obtained indicated that DXMS was effective in alleviating AA-induced rat vestibular dysfunction, and the activation of BDNF and inhibition of JNK1/2 singling pathways were the underlying mechanism. Further investigations of DXMS in human vestibular compensation are required.

\section{Materials and methods}

Ratbehavioral observation. A total of 30 male Sprague-Dawley rats (6-8 weeks old; weighing 200-250 g) were purchased from Jrdun Biotechnology (Shanghai) Co., Ltd., Shanghai, China. The rats were kept at $21 \pm 2^{\circ} \mathrm{C}$ and $50 \%$ humidity with 12-h light/dark cycles for a week prior to treatment, with free access to food and water. Vestibular dysfunction was established in rats by intratympanic injection of AA $(100 \mathrm{mg} / \mathrm{ml}$; $0.05 \mathrm{ml}$; Aladdin Bio-Chem Technology Co., Ltd., Shanghai, China) (16). After 3 days, behavioral observation was performed for $1 \mathrm{~h}$, and vestibular scores were subsequently assessed through the following behavioral assessments.

Air righting reflex. A sponge was spread on a flat surface and the animals were placed in a supine position in the air $50 \mathrm{~cm}$ away above the sponge, following which the animal was dropped horizontally. Four-legged smooth landing on the ground was considered as positive (normal animal), whereas, the body or other regions landing first were considered vestibular dysfunction animals. The positive landing rate $(\%)$ of each rat in each group was recorded.

Contact righting reflex. The rats were placed in a syringe core tube in a supine position, following which the recovery time for position correction was recorded. Normal rats turned over their body position in a few seconds. However, rats with vestibular dysfunction exhibited difficultly in identifying the inverted position, and thus failed to flip, or turn over their body position with prolonged time.

Head tilt and swimming behavior rating. The rats were placed in the center of a water bottle. The swimming behavior of animals within 2 min was observed. The grading standards for rat behaviors were as follows: 0 points, no dysfunction (no head tilt, straight swimming, reaching the wall or climbing); 1 point, marginal dysfunction (no obvious head deviation, some circular swimming, not always reaching the wall); 2 points, moderate dysfunction (marginal head deviation, frequent circular swimming, not always reaching the wall); and 3 points, serious dysfunction (obvious head deviation, circular swimming, not reaching the wall).
Experimental groups. The rats were randomly divided into the following groups ( $n=6 /$ group): i) Rats received vehicle (physiological saline) and considered the sham group; ii) Rats injected with AA to successfully induce vestibular dysfunction, considered the AA group; ii) AA + DXMS (1 mg/kg/day) group rats were subjected to AA-induced vestibular dysfunction, and then received DXMS (Sigma-Aldrich; Merck $\mathrm{KGaA}$, Darmstadt, Germany) at a dose of $1 \mathrm{mg} / \mathrm{kg} /$ day for 3 days through intraperitoneal injection; iv) AA + DXMS ( $3 \mathrm{mg} / \mathrm{kg} / \mathrm{day}$ ) group rats were treated as for the AA + DXMS (1 $\mathrm{mg} / \mathrm{kg} / \mathrm{day}$ ) group, but were administered with DXMS (3 $\mathrm{mg} / \mathrm{kg} /$ day) following AA-induced vestibular dysfunction; v) AA + DXMS + RU486 group rats were treated as the AA + DXMS (3 mg/kg/day) group, but were additionally administered RU486 (Sigma-Aldrich; Merck KGaA) at a dose of $100 \mathrm{mg} / \mathrm{kg} /$ day through subcutaneous injection into the nape of the neck. On the fourth day, behavioral observations of the rats in each group were performed, followed by the vestibular score assay. Subsequently, the rats were sacrificed, and brain tissue was collected for the subsequent experiments.

Hematoxylin-eosin (HE) staining. A total of six rats in each group were sacrificed to undergo thoracotomy. A tube was inserted at ascending aorta, perfused with $4 \%$ paraformaldehyde and the brain tissue was removed. At $4 \mathrm{~mm}$ from the optic chiasm, coronal sections (4-mm) were archived, routinely fixed with $10 \%$ formalin for $48 \mathrm{~h}$ at room temperature, washed three times with PBS (3 min/wash), dehydrated, paraffin-embedded and subsequently cut into sections. The 4- $\mu \mathrm{m}$ sections underwent $\mathrm{HE}$ staining at room temperature for 30 min and an Olympus CX41 light microscope (Olympus Corporation, Tokyo, Japan; magnification, x200) was used for visualization.

Terminal deoxyribonucleotidyl transferase-mediated biotinylated dUTP nick-end labeling (TUNEL) analysis. Following deparaffinization in xylene and rehydration in 100, 95, 85 and $75 \%$ gradient alcohol and double distilled water for 3 min each time, brain tissue sections $(4-7 \mu \mathrm{m})$ placed on glass slides were incubated with $50 \mu 1$ TUNEL solution (Roche Diagnostics, Basel, Switzerland) at $37^{\circ} \mathrm{C}$ for $1 \mathrm{~h}$. Subsequent to washing three times in PBS (3 min/wash), the slides were stained using a DAB substrate kit (cat. no. FL-6001; Shanghai Long Island Biotech Co., Ltd.) for $5 \mathrm{~min}$ at $37^{\circ} \mathrm{C}$ followed by counterstaining with hematoxylin (cat. no. 714094; BASO Diagnostic, Inc.) to stain nuclei at $37^{\circ} \mathrm{C}$ for $10 \mathrm{~min}$, and finally mounted with neutral balsam (Beijing Solarbio Science \& Technology Co., Ltd., Beijing, China) at $37^{\circ} \mathrm{C}$ for another $24 \mathrm{~h}$.

Apoptosis-positive cells were characterized by brown-yellow granules in the nucleus. A light microscope Nikon Eclipse Ni-U (Nikon Corporation. Tokyo, Japan; magnification, $\mathrm{x} 200$ ) equipped with a Nikon Ds-Ri2 microscopy digital camera was used for visualization. The area of apoptosis-positive cells was calculated using Image Pro-Plus 6.0 software (Media Cybernetics, Inc., Rockville, MD, USA) in 10 randomly selected microscopic fields per specimen.

Immunohistochemistry (IHC) assay. Following blocking with $1 \%$ bovine serum albumin [Sangon Biotech (Shanghai) Co., Ltd., Shanghai, China] at room temperature for $30 \mathrm{~min}$, 
the slides were incubated with primary antibodies: Mouse anti-BDNF (1:10,000; cat. no. ab203573; Abcam, Cambridge, MA, USA), rabbit anti-TrkB (1:100; cat. no. ab18987; Abcam) and mouse anti-KCC2 (1:200; cat. no. NBP2-59337; Novus Biologicals, LLC, Littleton, CO, USA) for $1 \mathrm{~h}$ at room temperature, followed by horseradish peroxidase (HRP)-conjugated mouse anti-rabbit antibody (cat. no. D-3004; Shanghai Long Island Biotech Co., Ltd., Shanghai, China) for $20 \mathrm{~min}$ at $25^{\circ} \mathrm{C}$. The slides were stained using a DAB-containing substrate kit (cat. no. FL-6001; Shanghai Long Island Biotech Co., Ltd.) to develop a brown color followed by hematoxylin staining (cat. no. 714094; BASO Diagnostic, Inc., Wuhan, China) for 3 min at room temperature. A fluorescence microscope (Leica Microsystems, Inc., Buffalo Grove, IL, USA; magnification, $\mathrm{x} 200$ ) was used to calculate regions of BDNF-, TrKB- or KCC2-positive cells.

Reverse transcription-quantitative polymerase chain reaction $(R T-q P C R)$ analysis. The mRNA and protein expression levels of BDNF, TrkB, KCC2, cleaved-caspase 3, JNK1/2 and phosphorylated (p)-JNK1/2 were assessed by RT-qPCR and western blot analyses, respectively, according to a previous study (5). Total RNA from brain tissues was extracted using TRIzol ${ }^{\circledR}$ Reagent (cat. no. 1596-026; Invitrogen; Thermo Fisher Scientific, Inc.) and reverse transcribed using a cDNA synthesis kit (cat. no. K1622; Fermentas; Thermo Fisher Scientific, Inc.). mRNA expression levels of BDNF, TrkB, KCC2 and cleaved-caspase 3 were determined using a SYBR Green PCR kit (Thermo Fisher Scientific, Inc.) on an ABI Prism 7300 SDS Software (Applied Biosystems; Thermo Fisher Scientific, Inc.) with amplification condition programed as: $95^{\circ} \mathrm{C}$ for $10 \mathrm{~min}$; 40 cycles for $95^{\circ} \mathrm{C}$ for $15 \mathrm{sec} ; 60^{\circ} \mathrm{C}$ for $45 \mathrm{sec} ; 95^{\circ} \mathrm{C}$ for $15 \mathrm{sec}$; $60^{\circ} \mathrm{C}$ for $1 \mathrm{~min} ; 95^{\circ} \mathrm{C}$ for $15 \mathrm{sec}$; and $60^{\circ} \mathrm{C}$ for $15 \mathrm{sec}$. mRNA expression levels of GAPDH served as an internal control. Expression was calculated using the $2^{-\Delta \Delta \mathrm{Cq}}$ method (17). The primer pairs targeting BDNF (GenBank NM_001270630.1), TrkB (GenBank NM_001163168.2), KCC2 (GenBank NM_ NM_134363.1), cleaved-caspase 3 (GenBank NM_012922.2) and an internal standard GAPDH were as follows: BDNF, forward 5'-GACAAGGCAACTTGGCCTAC-3' and reverse 5'-TCCAGCAGCTCTTCGATCAC-3' (position: 452-590; product size: $139 \mathrm{bp}$ ); TrkB, forward 5'-ACTGGACCACGC CAACTGAC-3' and reverse 5'-TCACCACCACGGCATAGA CC-3' (position: 1872-1988; product size: 117 bp); KCC2, forward 5'-ATCGAGATCCTGCTGGCTTAC-3' and reverse 5'-CTTGACGCCCACAAAGACTAC-3' (position: 713-865; product size: $153 \mathrm{bp}$ ); cleaved-caspase 3, forward 5'-GGC ATCTCCTGTGATTGG-3' and reverse 5'-CTCAGCACT CTGGGAAAG-3' (position: 1780-1964; product size: 185 bp); GAPDH, forward 5'-GGAGTCTACTGGCGTCTTCAC-3' and reverse 5'-ATGAGCCCTTCCACGATGC-3' (position: 357-593; product size: $237 \mathrm{bp}$ ).

Western blot analysis. A total of $30 \mu \mathrm{g}$ total protein underwent western blot analysis. Following full lysis of brain tissue $(20 \mathrm{mg})$ in radioimmunoprecipitation assay buffer (Jrdun Biotechnology (Shanghai) Co., Ltd.), samples were centrifuged $\left(12,000 \mathrm{x} \mathrm{g} ; 10 \mathrm{~min} ; 4^{\circ} \mathrm{C}\right)$. Total protein content in the supernatant was determined using a Bicinchoninic Acid Protein Assay kit (Beyotime Institute of Biotechnology,
Haimen, China). In total, $30 \mu \mathrm{g}$ total protein in the supernatant was separated using 15\% SDS-PAGE, and electrophoretically pure of BDNF, TrkB, KCC2, cleaved-caspase 3, JNK1/2 and $\mathrm{p}-\mathrm{JNK} 1 / 2$ were transferred onto nitrocellulose (NC) membranes (cat. no. HATF0001; EMD Millipore, Billerica, MA, USA). Subsequent to blocking with $5 \%$ nonfat milk at room temperature for $1 \mathrm{~h}, \mathrm{NC}$ membranes were incubated with antibody against BDNF (1:300 dilution; cat. no. ab203573; Abcam), antibody against TrKB (1:500; cat. no. ab18987; Abcam), antibody against KCC2 (1:200; cat. no. NBP2-59337; Novus Biologicals, LLC), antibody against caspase 3 (1:500; cat no. ab44976; Abcam), antibody against JNK1/2 (1:1,000; cat. no. 9252; Cell Signaling Technology, Inc., Danvers, MA, USA), antibody against p-JNK1/2 (1:1,000; cat. no. 9255; Cell Signaling Technology, Inc.) and antibody against GAPDH (1:2,000; cat. no. 5174; Cell Signaling Technology, Inc.) at $4^{\circ} \mathrm{C}$ overnight followed by incubation with HRP-conjugated secondary antibody (1:1,000; Beyotime Institute of Biotechnology) at $37^{\circ} \mathrm{C}$ for $1 \mathrm{~h}$. Immunoreactive bands were analysed using an enhanced chemiluminescent system (GE Healthcare, Chicago, IL, USA) with ImageJ software (Version 1.6; National Institutes of Health, Bethesda, MD, USA). Protein expression levels of BDNF, TrKB, KCC2, caspase 3 and JNK1/2 were normalized using GAPDH, whereas, p-JNK1/2 was normalized using JNK1/2.

Statistical analysis. GraphPad Prism 7 software (GraphPad Software, Inc., La Jolla, CA, USA) was used for graphing. Values are presented as the mean \pm standard deviation $(n=3)$. Significant differences between groups were calculated using one-way analysis of variance with Tukey's post-hoc test. $\mathrm{P}<0.05$ was considered to indicate a statistically significant difference. For IHC analysis, the areas of BDNF-, TrkBand KCC2-positive cells were detected in five high-power fields (HPF; magnification, x200). The results based on the percentage of stained cells were graded as weakly positive $(<25 \%)$, medium positive $(25-49 \%)$ and strongly positive $(>50 \%)$. For TUNEL analysis, the percentage of apoptotic cells were measured in 10 HPF (magnification, x200), which were graded as scores of $0(<10 \%), 1(10-25 \%), 2(26-50 \%)$ and $3(>50 \%)$. The degree of staining was divided into scores of 0 (none), 1 (light yellow), 2 (brown yellow) and 3 (brown). The results based on the addition of those two criteria were considered as positive ( $\geq 4$ score) and negative ( $<4$ score).

\section{Results}

Successful establishment of vestibular dysfunction. At 3 days following the administration of AA $(98 \% ; 100 \mathrm{mg} / \mathrm{ml})$, the rats in the AA group manifested classic symptoms of vestibular injury, including dizziness, nausea, vomiting, nystagmus, head-shaking nystagmus, head tilt and postural instability.

In the air righting reflex test (Fig. 1A), the positive rate of the AA-treated group was $\sim 25 \pm 2 \%$, which was significantly lower compared with the control group $(87 \pm 1 \%)$. In the contact righting reflex test (Fig. 1B), the rats in the sham group returned from a supine position to a normal posture within $5 \mathrm{sec}$; however, the rats in the AA group took $30-50 \mathrm{sec}$, indicating a significant difference in the righting reflex time between the two groups $(\mathrm{P}<0.01)$. In the head tilt and swimming behavior 

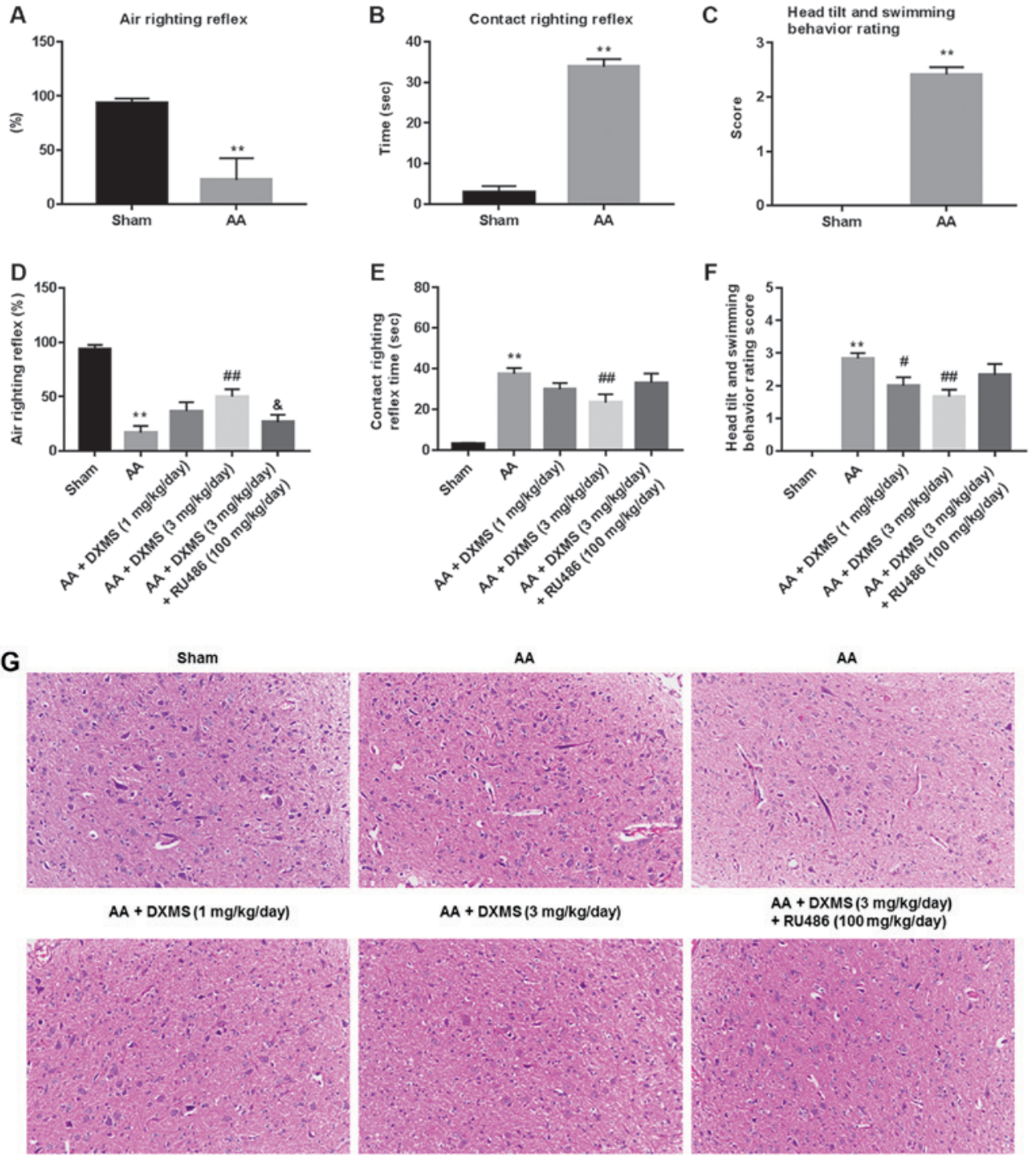

$A A+D X M S$ ( 3 mg/kg/day)

$\mathrm{AA}+\mathrm{DXMS}(3 \mathrm{mg} / \mathrm{kg} /$ day $)$
$+\mathrm{RU} 486(100 \mathrm{mg} / \mathrm{kg} /$ day $)$
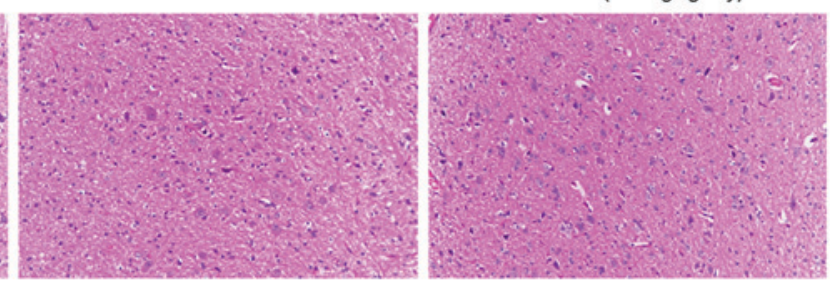

H

Sham

AA

$\mathrm{AA}+\mathrm{DXMS}(1 \mathrm{mg} / \mathrm{kg} /$ day)
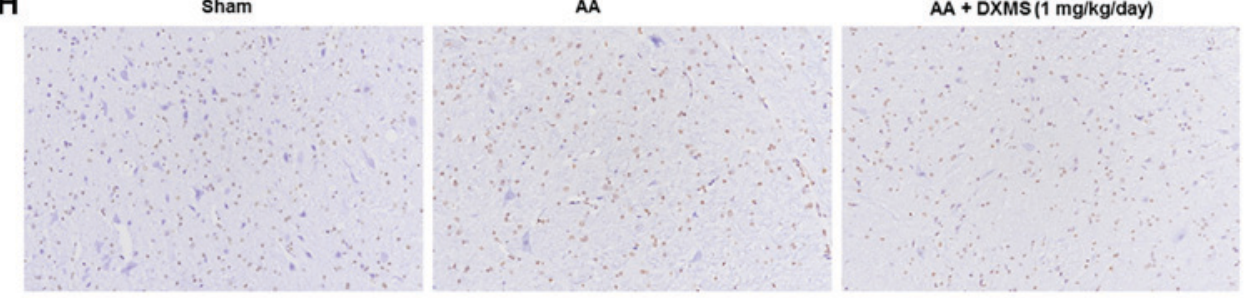

$A A+D X M S(3 \mathrm{mg} / \mathrm{kg} /$ day)

AA + DXMS (3 mg/kg/day)
+ RU486 (100 mg/kg/day)
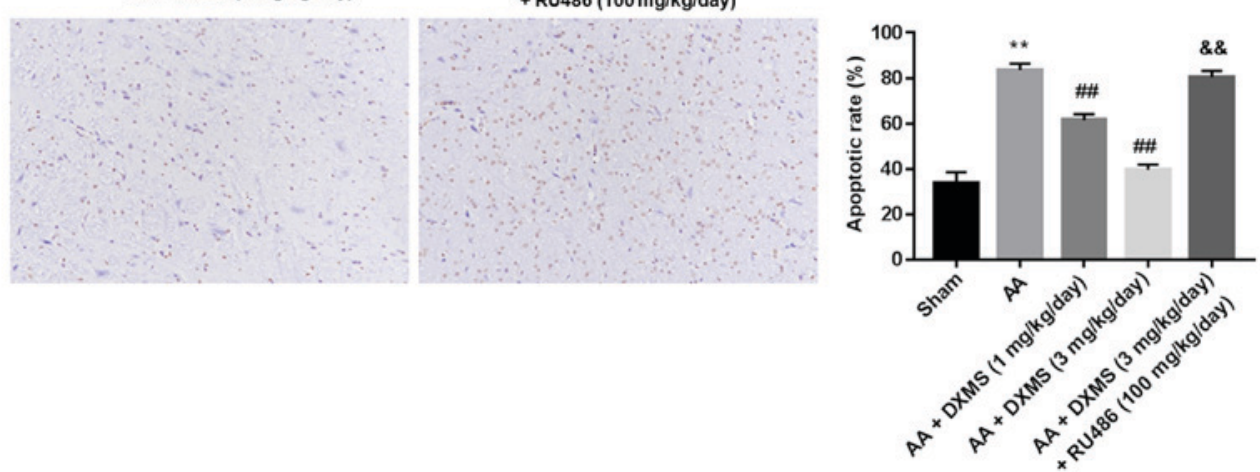

Figure 1. DXMS is effective in recovering from AA-induced rat vestibular dysfunction. A total of 30 rats were randomly divided into a sham group (n=6) and AA group (n=24). (A) Air righting reflex, (B) contact righting reflex and $(\mathrm{C})$ head tilt and swimming behavior rating demonstrated successful construction of AA-induced rat vestibular dysfunction. DXMS improved behavior scores in AA-treated rats, as determined by (D) air righting reflex, (E) contact righting reflex and $(\mathrm{F})$ head tilt and swimming behavior rating. $(\mathrm{G})$ Histopathological and $(\mathrm{H})$ terminal deoxyribonucleotidyl transferase-mediated biotinylated dUTP nick-end labeling analyses (magnification, $\mathrm{x} 200$ ) indicated that DXMS reduced brain damages in the $\mathrm{AA}$-treated rats. ${ }^{* *} \mathrm{P}<0.01$ vs. sham group; ${ }^{*} \mathrm{P}<0.05$, ${ }^{\# \#} \mathrm{P}<0.01$ vs. AA group; ${ }^{\&} \mathrm{P}<0.05,{ }^{\& \&} \mathrm{P}<0.01$ vs. AA + DXMS (3 mg/kg/day). DXMS, dexamethasone; AA, arsanilic acid. 

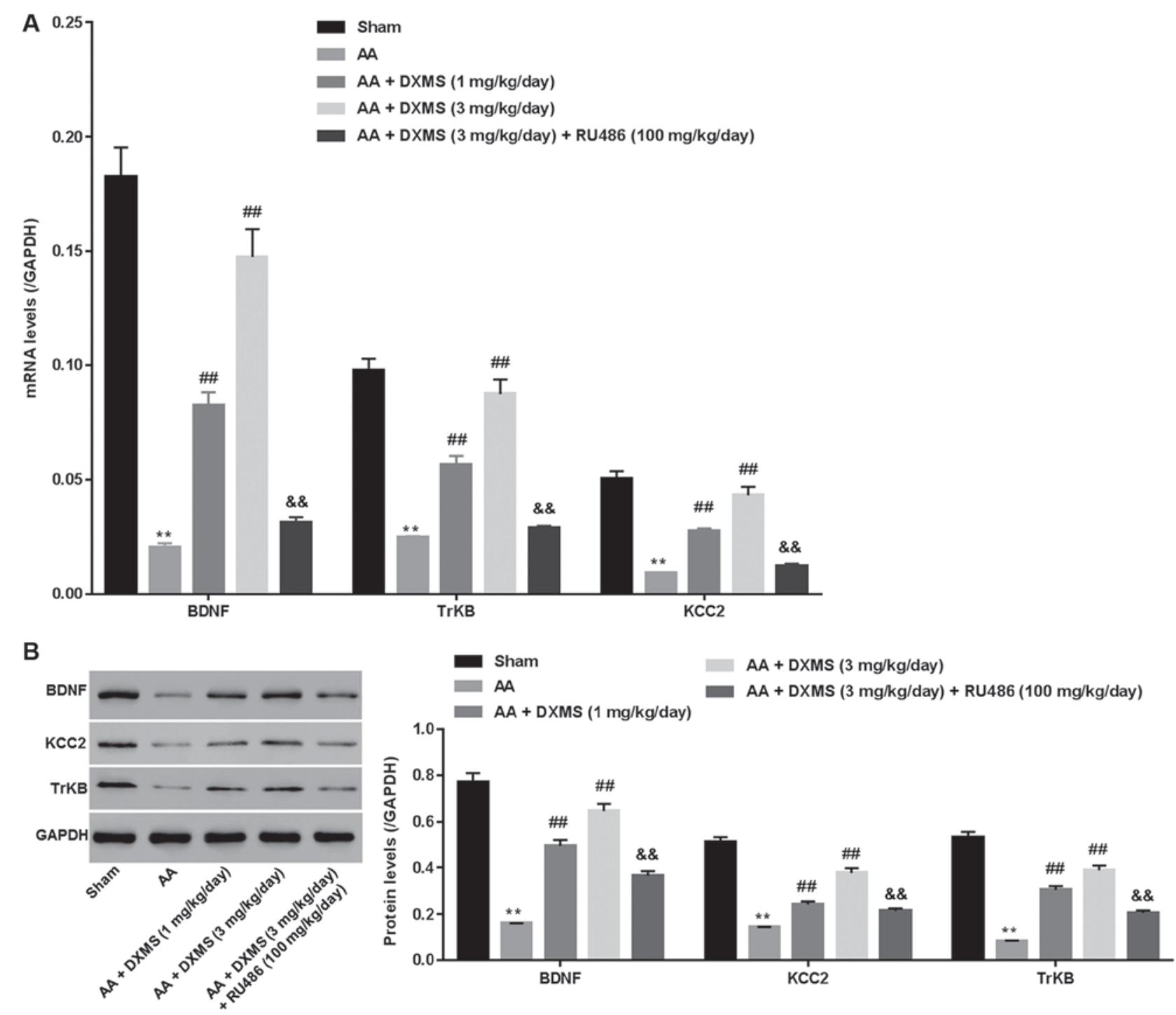

Figure 2. DXMS activates the BDNF signaling pathway in AA-treated rats. (A) Reverse transcription-quantitative polymerase chain reaction analysis results demonstrating mRNA levels of BDNF, TrkB and $\mathrm{KCC} 2$ in brain tissues of each groups. (B) Western blotting demonstrating protein expression levels of BDNF, TrkB and KCC2 in brain tissues of each group. Each experiment was repeated three times. ${ }^{* *} \mathrm{P}<0.01$ vs. sham group; ${ }^{\# / t} \mathrm{P}<0.01 \mathrm{vs}$. AA group; \&\& $\mathrm{P}<0.01 \mathrm{vs}$. AA + DXMS (3 mg/kg/day) group. DXMS, dexamethasone; AA, arsanilic acid; BDNF, brain-derived neurotrophic factor; TrkB, tyrosine receptor kinase B; $\mathrm{KCC} 2, \mathrm{~K}^{+} / \mathrm{Cl}^{-}$cotransporter isoform 2 .

rating test (Fig. 1C), the score of the AA group (score of 3) was significantly higher compared with the sham group (score of 0 ). Overall, the symptom observation and behavioral investigations suggested the successful establishment of AA-induced rat vestibular dysfunction.

DXMS is a therapeutic drug for AA-induced rat vestibular dysfunction. Syndromes accompanied with vestibular dysfunction in the A A + DXMS ( $1 \mathrm{mg} / \mathrm{kg} / \mathrm{day}$ ) group were marginally alleviated, with significant improvements in terms of gradual disappearance of head tilt, postural instability and nystagmus in the AA + DXMS (3 mg/kg/day) group. DXMS moderately increased scores in the air righting reflex test, reduced the times in the contact righting reflex test and decreased scores in the head tilt and swimming behavior rating test, however, with additional RU486 treatment, the DXMS-induced disappearance on symptoms of vestibular dysfunction and improvement of vestibular scores were significantly reversed (Fig. 1D-F).
DXMS reduces damage to brain tissues in AA-treated rats. In the histopathological analysis (Fig. 1G), the AA group exhibited disorganized neuron arrangement, widened extracellular spaces, retracted nuclei, and disappearance of nucleoli when compared with the corresponding sham group, demonstrating severe damage in the brain tissue. In the DXMS-treated groups (1 and $3 \mathrm{mg} / \mathrm{kg} / \mathrm{day}$ ), the extracellular spaces and nuclear condensation were significantly reduced in a dose-dependent manner, and the cytoplasm and nucleus were clearer when compared with those in the AA group, demonstrating that DXMS reduced damage to the brain tissues in the AA-treated rats. In the TUNEL analysis (Fig. $1 \mathrm{H}$ ), the apoptotic area (\%) of the brain tissue in the AA group was larger $(83.5 \pm 1 \%)$ compared with the sham group $(33.5 \pm 1.7 \%)$. However, DXMS significantly decreased the apoptotic area in a dose-dependent manner with a minimum value $(39.6 \pm 0.8 \%)$ observed at $3 \mathrm{mg} / \mathrm{kg} / \mathrm{day}$. The DXMS-induced histopathological and apoptotic changes were significantly reversed with additional 

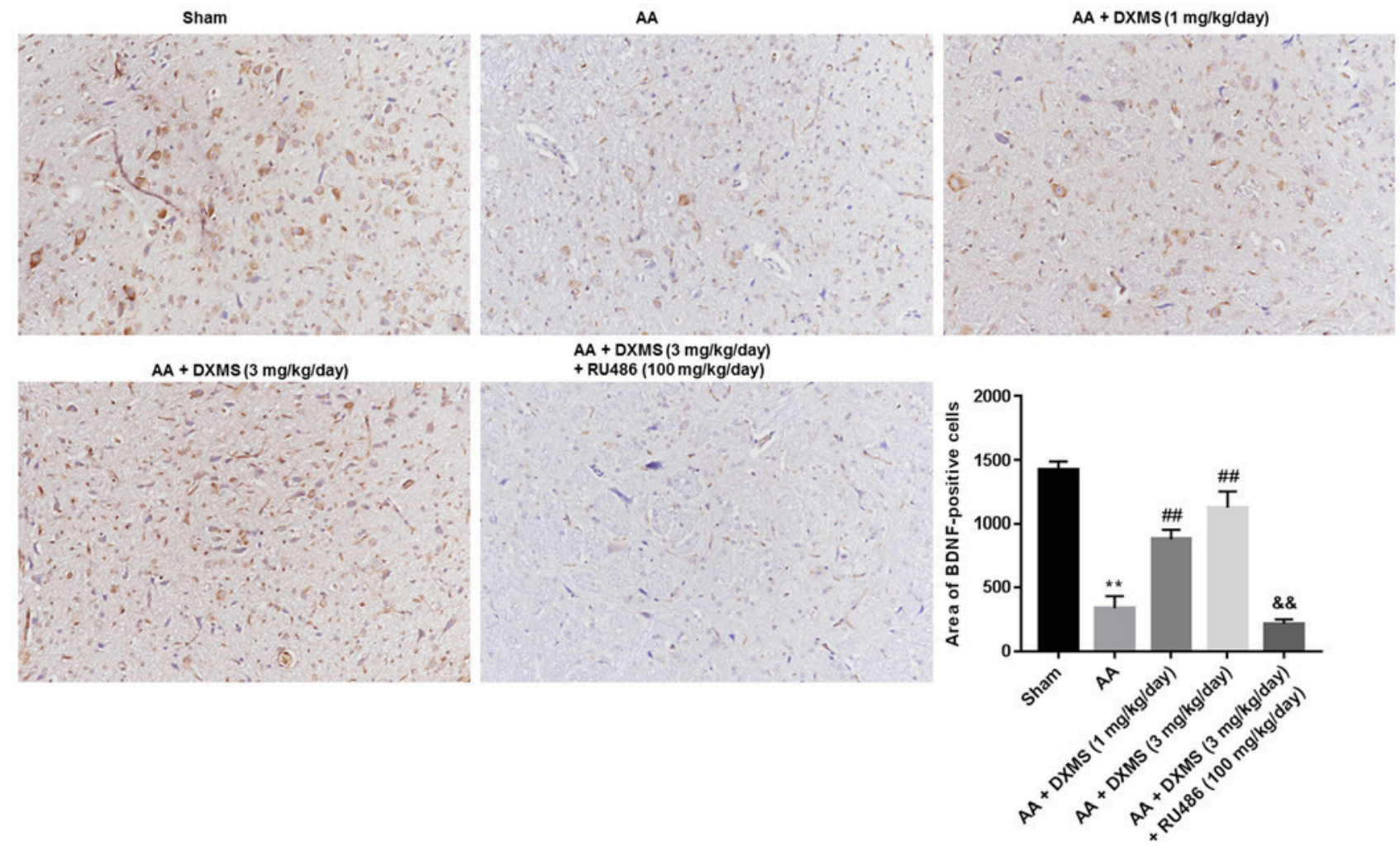

Figure 3. DXMS enhances BDNF-positive cells. Immunohistochemical staining of BDNF demonstrated that DXMS effectively enhanced areas of BDNF-positive cells in brain tissues of AA-treated rats (magnification, $\mathrm{x} 200$ ). ${ }^{* *} \mathrm{P}<0.01$ vs. normal primary cells; ${ }^{* *} \mathrm{P}<0.01$ vs. sham group; ${ }^{\# \#} \mathrm{P}<0.01 \mathrm{vs}$. AA group; \&\& $\mathrm{P}<0.01$ vs. AA + DXMS (3 mg/kg/day) group. DXMS, dexamethasone; AA, arsanilic acid; BDNF, brain-derived neurotrophic factor.

RU486 treatment, suggesting that RU486 was an inhibitor of the therapeutic effect of DXMS on AA-induced rat vestibular dysfunction.

DXMS activates the BDNF signaling pathway in AA-treated rats. Activating the BDNF signaling pathway contributed to vestibular compensation. To examine whether the BDNF signaling pathway was involved in the protective effect of DXMS on AA-induced vestibular dysfunction, BDNF signaling pathway-associated BDNF, TrkB and KCC2 were assessed. As presented in Fig. 2A and B, significantly decreased mRNA and protein levels of BDNF, TrkB and KCC2 were obtained in the AA group when compared with the sham group $(\mathrm{P}<0.01)$, confirming that inhibition of the BDNF signaling pathway was involved in AA-induced vestibular dysfunction. By contrast, DXMS significantly increased the mRNA and protein expression levels of BDNF, TrkB and KCC2 in a dose-dependent manner, the alterations of which were markedly reversed following additional RU486 treatment. This suggested that activating the BDNF signaling pathway was the mechanism by which DXMS exerted its protective effect on AA-induced vestibular dysfunction.

The expression levels and distribution of BDNF, TrkB and $\mathrm{KCC} 2$ in the brain sections were separately determined by an IHC assay. As demonstrated in Fig. 3, similar to the mRNA and protein expression levels of BDNF, the areas of BDNF-positive cells were significantly decreased in the AA group when compared with the sham group. DXMS enhanced the areas of BDNF-positive cells in a dose-dependent manner, and reached a peak value at $3 \mathrm{mg} / \mathrm{kg} / \mathrm{day}$, which was significantly decreased with additional RU486 treatment $(\mathrm{P}<0.01)$.
As presented in Figs. 4 and 5, similar trends of change were observed in the areas of TrkB or KCC2-positive cells.

DXMS decreases cleaved-caspase 3 and inhibits the JNK pathway in AA-treated rats. The JNK signaling pathway is essential for triggering apoptosis in brain tissues following a variety of injuries (18). Cleaved-caspase 3 is known as a key pro-apoptotic mediator. To assess the anti-apoptotic mechanism of DXMS in AA-treated rats, cleaved-caspase 3 and the JNK signaling pathway were detected. It was identified that, in the AA group, a marked increase in the mRNA level of cleaved-caspase 3 (Fig. 6A) and the protein expression levels of cleaved-caspase 3 and p-JNK1/2 (Fig. 6B and C) were observed when compared with those in the sham group, and these alterations were significantly reversed with DXMS treatment in a dose-dependent manner. In the AA + DXMS (3 $\mathrm{mg} / \mathrm{kg} / \mathrm{day})+$ RU486 group, the expression levels of cleaved-caspase 3 and p-JNK1/2 were markedly enhanced when compared with those of the AA + DXMS (3 mg/kg/day) group $(\mathrm{P}<0.01)$. There was approximately no distinguished difference in the protein expression levels of JNK1/2 in the groups. These data suggested that reducing the expression of cleaved-caspase 3 and preventing activation of the JNK1/2 pathway were the anti-apoptotic mechanisms underlying the effect of DXMS in AA-induced vestibular dysfunction.

\section{Discussion}

In the present study, rats were treated with AA at a dose of $100 \mathrm{mg} / \mathrm{ml}$ to establish a rat vestibular lesioning model, which 

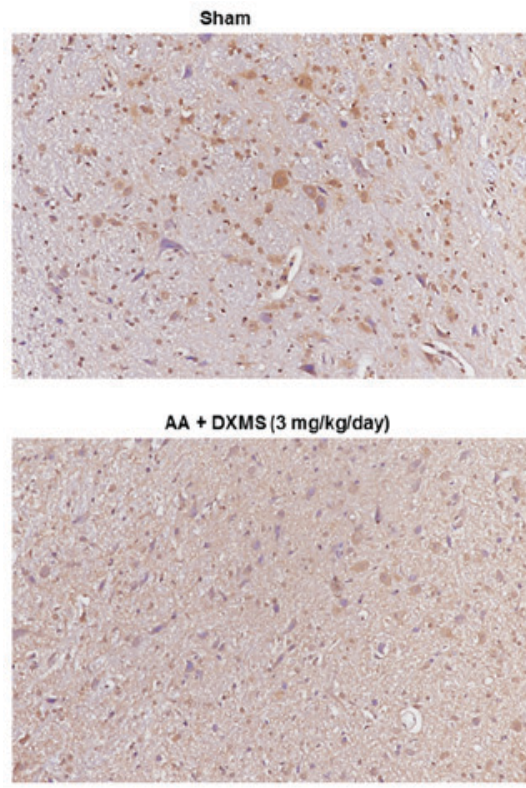

AA

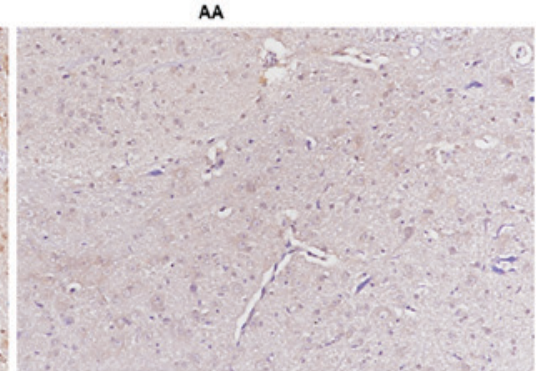

$\mathrm{AA}+\mathrm{DXMS}(3 \mathrm{mg} / \mathrm{kg} /$ day $)$

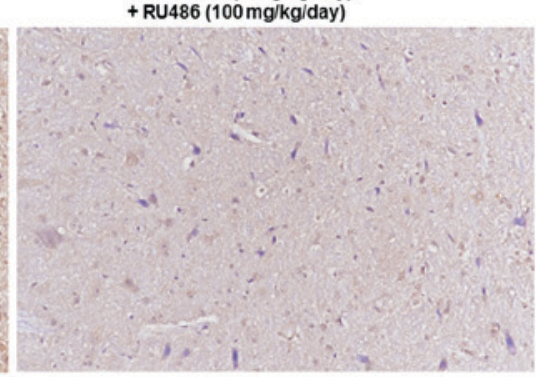

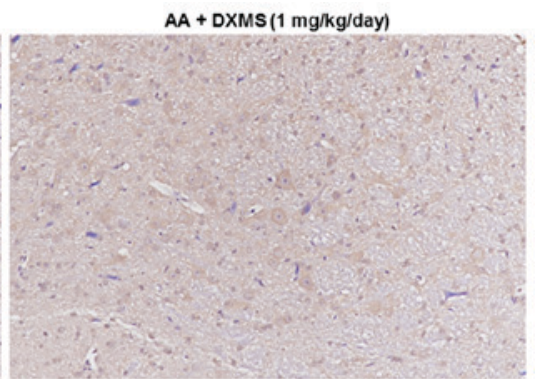

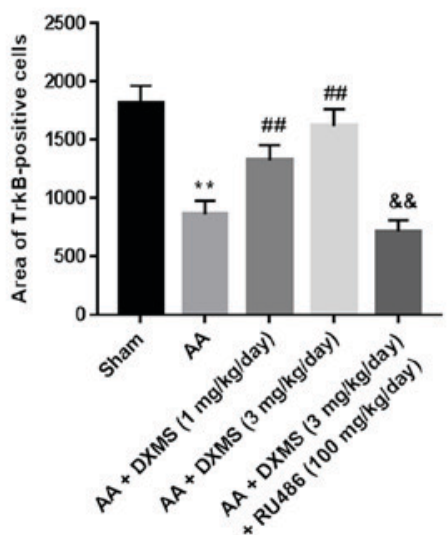

Figure 4. DXMS enhances TrkB-positive cells. Immunohistochemical staining of TrkB demonstrated that DXMS effectively increased areas of TrkB-positive cells in brain tissues of AA-treated rats (magnification, $\mathrm{x} 200$ ). ${ }^{* *} \mathrm{P}<0.01$ vs. sham group; ${ }^{\# /} \mathrm{P}<0.01 \mathrm{vs}$. AA group; \&\& $\mathrm{P}<0.01 \mathrm{vs}$. AA $+\mathrm{DXMS}(3 \mathrm{mg} / \mathrm{kg} / \mathrm{day}$ ) group. DXMS, dexamethasone; AA, arsanilic acid; TrkB, tyrosine receptor kinase B.
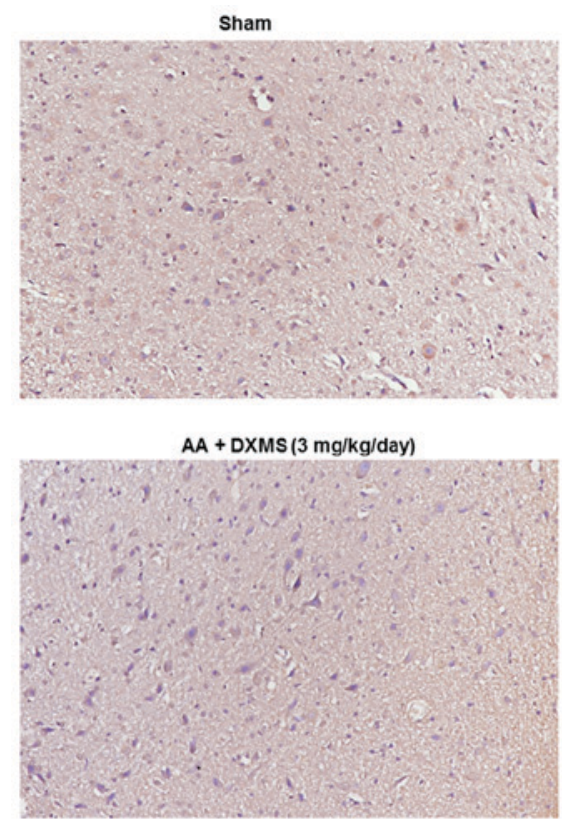

AA

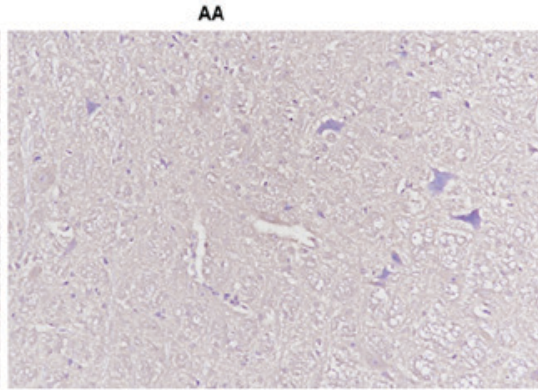

$\mathrm{AA}+\mathrm{DXMS}(3 \mathrm{mg} / \mathrm{kg} /$ day $)$

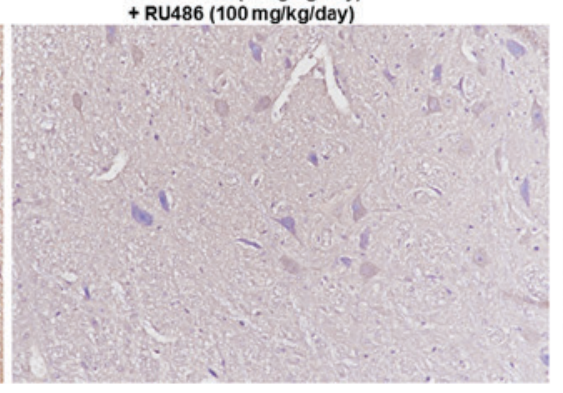

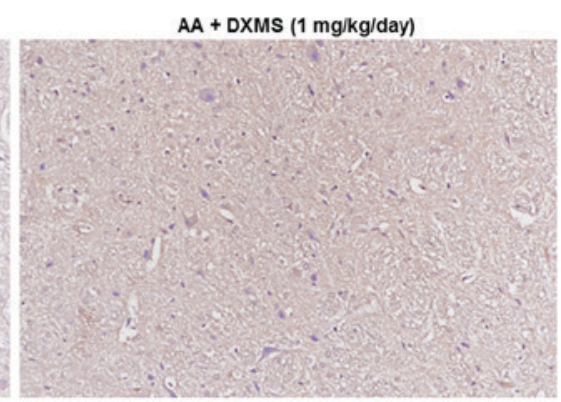

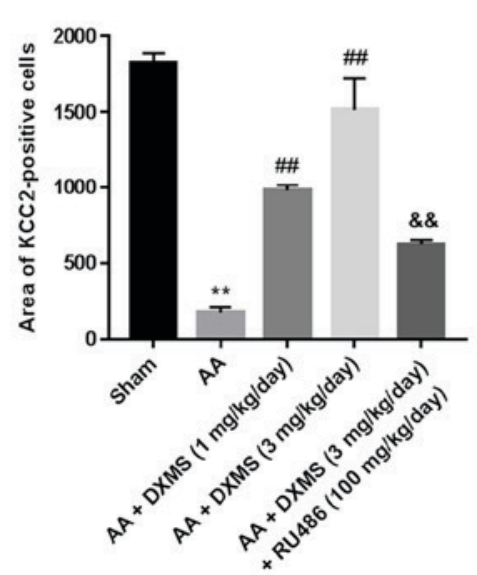

Figure 5. DXMS enhances KCC2-positive cells Immunohistochemical staining of KCC2 demonstrated that DXMS effectively enhanced areas of KCC2-positive cells in brain tissues of AA-treated rats (magnification, $\mathrm{x} 200){ }^{* * *} \mathrm{P}<0.01 \mathrm{vs}$. sham group; ${ }^{\# \#} \mathrm{P}<0.01 \mathrm{vs}$. AA group; \& ${ }^{\star} \mathrm{P}<0.01 \mathrm{vs}$. AA + DXMS (3 mg/kg/day) group. DXMS, dexamethasone; AA, arsanilic acid; $\mathrm{KCC} 2, \mathrm{~K}^{+} / \mathrm{Cl}^{-}$cotransporter isoform 2.

was confirmed by observing syndromes that were associated with vestibular dysfunction, including low-positive rates in the air righting reflex test, prolonged times in the contact righting reflex test, and high scores in the head tilt and swimming 
A
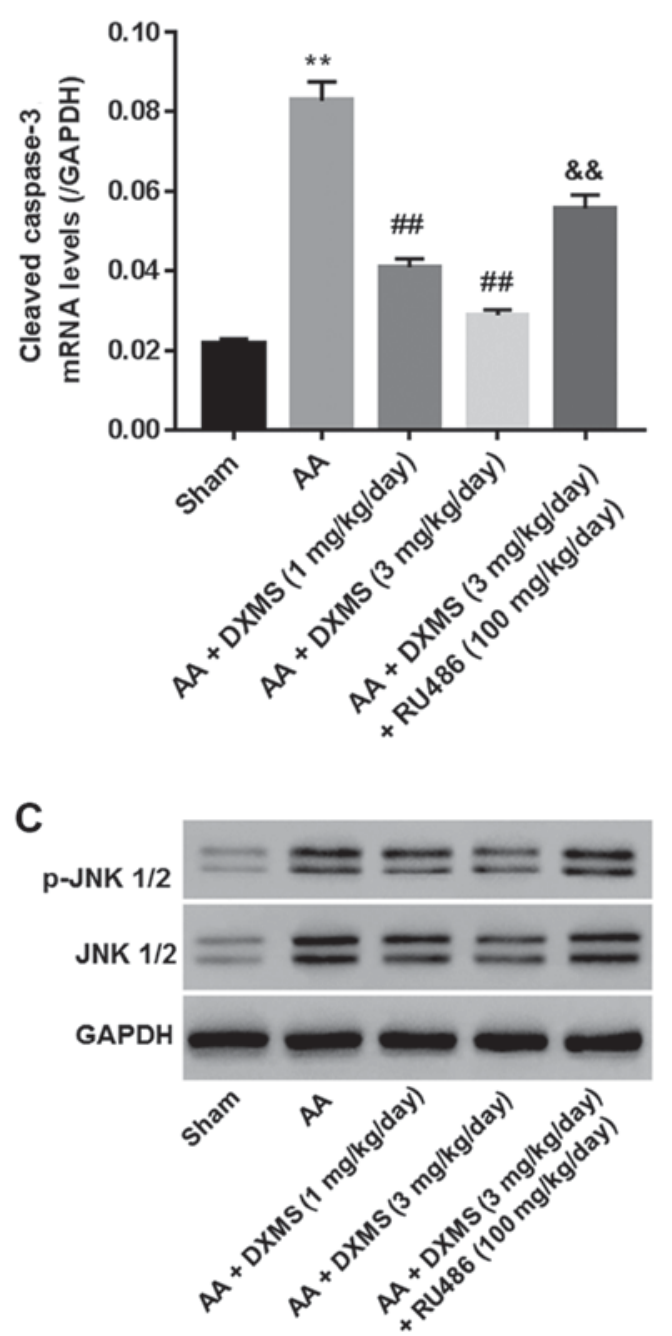

B

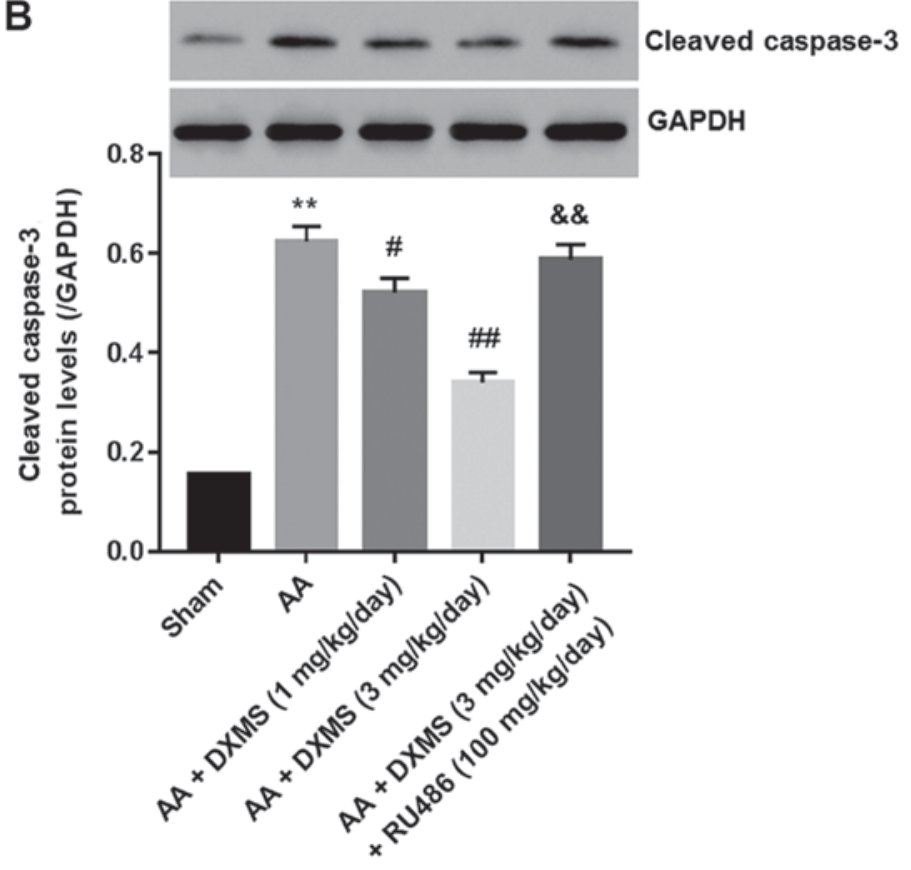

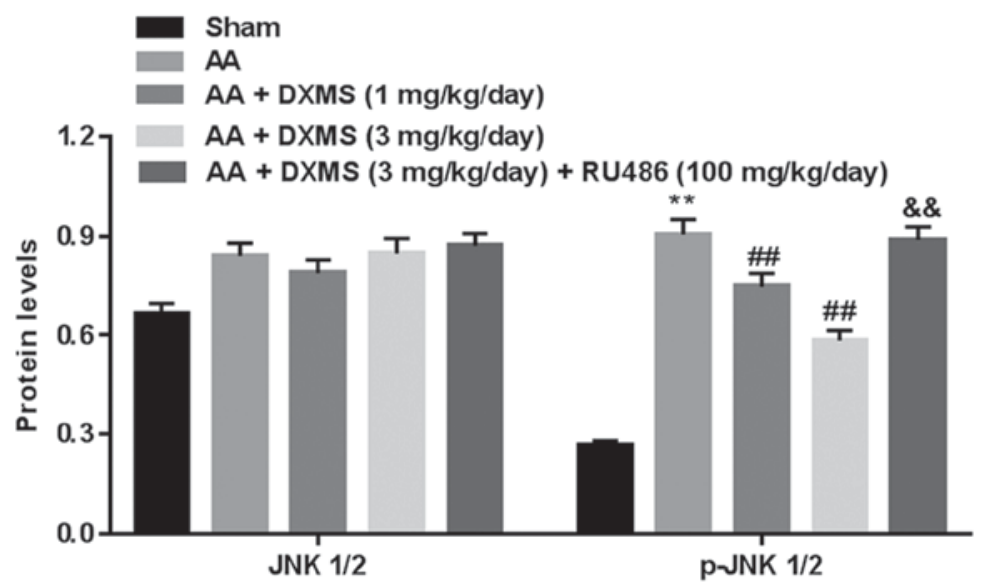

Figure 6. DXMS prevents JNK1/2 signaling pathways in AA-treated rats. (A) Protein and (B) mRNA expression levels of cleaved-caspase 3 in brain tissues. (C) Protein expression levels of JNK1/2 and p-JNK1/2. Each experiment was repeated three times. ${ }^{* *} \mathrm{P}<0.01 \mathrm{vs}$. sham group; ${ }^{\#} \mathrm{P}<0.05$, ${ }^{\# \#} \mathrm{P}<0.01 \mathrm{vs}$. AA group; ${ }^{\&}$ P $<0.01$ vs. AA + DXMS (3 mg/kg/day) group. DXMS, dexamethasone; AA, arsanilic acid; JNK, c-Jun NH 2-terminal kinase; p-JNK, phosphorylated JNK.

behavior test. The histopathological evaluation and TUNEL analysis demonstrated severe brain damage, and increased areas of apoptotic cells in brain tissues, further substantiating vestibular deficits in the AA-treated rats.

At present, DXMS, a known glucocorticoid, has been reported to have a neuroprotective effect on hypoxia/ischemia-stimulated, or traumatic brain injury (19-21). In 1995, Yamanaka et al (15) reported that DXMS directly activates the medial vestibular nucleus, thus accelerating vestibular compensation. However, whether and how DXMS exerts its protective effect on AA-induced vestibular lesions remains to be fully elucidated. Intraperitoneal injection of dexamethasone into rats at a dose of $1 \mathrm{mg} / \mathrm{kg} /$ day has been used to treat traumatic brain injury, therefore, in the present study, doses of 1 and $3 \mathrm{mg} / \mathrm{kg} / \mathrm{day}$ were selected for the investigation (22). It was identified that, with DXMS treatment, AA-induced rat vestibular dysfunction was significantly alleviated in a dose-dependent manner, demonstrated by the disappearance in classic signs of rat vestibular injury, improved behavior scores, minor damage and decreased apoptotic cells in brain tissues. In addition, RU486 serves as a glucocorticoid receptor antagonist and is usually subcutaneously injected into rats at a dose of $10 \mathrm{mg} / \mathrm{kg} / \mathrm{day}$; this which was additionally adopted in the present experiment proposal for DXMS therapeutic intervention $(23,24)$. The resulting data suggested that RU486 significantly worsened vestibular symptoms and behavior scores, and reversed DXMS-induced histopathologic changes in brain tissues, demonstrating that RU484 inhibited vestibular compensation following DXMS treatment. Overall, the data indicated that DXMS was an effective drug for recovery of AA-induced rat vestibular dysfunction.

BDNF-triggered activation of TrkB reduces the expression of KCC2, a specific marker of neuronal excitability (25). At present, the $\mathrm{BDNF} / \mathrm{TrkB} / \mathrm{KCC} 2$ pathway is known to be required for the development of the vestibular system, and additionally for behavioral recovery following vestibular dysfunction in adult mammals. Therefore, the BDNF signaling pathway is widely 
recognized as a therapeutic target for vestibular compensation (7). The present study further investigated whether BDNF signaling pathway was the mechanism that DXMS exerted its protective effect on AA-induced rat vestibular dysfunction. It was identified that AA significantly decreased the mRNA and protein expression levels of BDNF, TrkB and $\mathrm{KCC} 2$, and the positive cells rates of BDNF, TrkB and $\mathrm{KCC} 2$ (as determined by IHC staining) in brain tissues, confirming that inhibition of the BDNF singling pathway was involved in AA-induced vestibular dysfunction. However, DXMS markedly up-regulated BDNF, TrkB and KCC2 in AA-induced rat vestibular dysfunction, the effect of which was suppressed with additional RU484 treatment. This demonstrated that DXMS confirmed AA-induced rat vestibular dysfunction through the BDNF signaling pathway.

Caspases are principal executioners of various apoptotic pathways (26). Evidence indicates that the JNK pathway is involved in cell loss in the central nervous system (27). In 2009, Shuto et al (28) reported the anti-apoptotic effect of DXMS by inhibiting caspase-3 and retarding the JNK pathway in trimethyltin-induced neuronal damage. The present study investigated whether cleaved-caspase 3 and JNK1/2 signals were involved in the anti-apoptotic mechanism of DXMS in brain tissues of AA-stimulated rats. Firstly, it was confirmed that AA triggered the activation of cleaved-caspase 3 and the JNK1/2 pathway in brain tissues of rats with vestibular dysfunction. Secondly, it was found that DXMS significantly inhibited the mRNA expression of caspase-3, and suppressed the protein levels of caspase- 3 and p-JNK1/2 in the AA-treated rats, and these alterations were approximately entirely reversed by treatment with RU484. These data indicated that suppressing the transcription and translation of cleaved-caspase $3 \mathrm{mRNA}$, and inhibiting the JNK1/2 signaling pathway were involved in the anti-apoptotic effect of DXMS in AA-treated rats.

In conclusion, DXMS demonstrated a therapeutic effect against AA-induced dysfunction vestibular. The underlying mechanisms involved activating BDNF and inhibiting JNK1/2 signaling pathways. In vitro experiments are required to confirm this conclusion, and experiment materials are being prepared in our laboratory. Unfortunately, the results are unavailable at this point.

\section{Acknowledgements}

Not applicable.

\section{Funding}

The present study was supported by the Guidance Projects for Science and Technology Development Plan of Suzhou (grant no. SYSD2013086; China).

\section{Availability of data and materials}

The datasets used and/or analysed during the present study are available from the corresponding author on reasonable request.

\section{Authors' contributions}

YL conducted the experiment and made data entry. DZ conducted the statistical analysis and interpreted the data.
Data acquisition, analysis and interpretation were performed by $\mathrm{YC}$ and ZC. ZF designed the study.

\section{Ethics approval and consent to participate}

All experimental procedures involving rats followed the local committee on the ethical use of animals of Shandong Provincial Hospital (Jinan, China). The study was approved by the Institutional Review Board of Shandong Provincial Hospital Affiliated to Shandong University.

\section{Patient consent for publication}

Not applicable.

\section{Competing interests}

The authors declare that they have no competing interests.

\section{References}

1. Cullen K and Sadeghi S: Vestibular system. Scholarpedia 3: 2008.

2. Borel L, Lopez C, Péruch P and Lacour M: Vestibular syndrome: A change in internal spatial representation. Neurophysiol Clin 38: 375-389, 2008

3. Hotson JR and Baloh RW: Acute vestibular syndrome. N Engl J Med 339: 680-685, 1998.

4. Wu X, Cai J, Li X, Li H, Li J, Bai X, Liu W, Han Y, Xu L, Zhang D, et al: Allicin protects against cisplatin-induced vestibular dysfunction by inhibiting the apoptotic pathway. Eur J Pharmacol 805: 108-117, 2017.

5. Zhou L, Zhou W, Zhang S, Liu B, Liang P,Zhou Y, Zhou T, Zhang K, Leng $\mathrm{Y}$ and Kong W: BDNF signaling in the rat cerebello-vestibular pathway during vestibular compensation: BDNF signaling in vestibular compensation. FEBS J 282: 3579-3591, 2015.

6. Tyler WJ and Pozzo-Miller L: Miniature synaptic transmission and BDNF modulate dendritic spine growth and form in rat CA1 neurones. J Physiol 553: 497-509, 2003.

7. Dutheil S, Watabe I, Sadlaoud K, Tonetto A and Tighilet B: BDNF signaling promotes vestibular compensation by increasing neurogenesis and remodeling the expression of potassium-chloride cotransporter $\mathrm{KCC} 2$ and GABAA receptor in the vestibular nuclei. J Neurosci 36: 6199-6212, 2016.

8. Botelho F, Lai C, Shum D and Chan Y: BDNF-mediated GABAergic transmission in the rat vestibular nucleus regulates the developmental recognition of spatial orientation. The 2012 Annual Meeting of the Society for Neuroscience: 735.11/A55, 2012.

9. Willson ML, Mcelnea C, Mariani J, Lohof AM and Sherrard RM BDNF increases homotypic olivocerebellar reinnervation and associated fine motor and cognitive skill. Brain 131: 1099-1112, 2008.

10. Gómez-Casati ME, Murtie JC, Rio C, Stankovic K, Liberman MC and Corfas G: Nonneuronal cells regulate synapse formation in the vestibular sensory epithelium via erbB-dependent BDNF expression. Proc Natl Acad Sci USA 107: 17005-17010, 2010.

11. He YY, Zhang XY, Yung WH, Zhu JN and Wang JJ: Role of BDNF in central motor structures and motor diseases. Mol Neurobiol 48: 783-793, 2013.

12. Trune DR and Canlon B: Corticosteroid therapy for hearing and balance disorders. Anat Rec (Hoboken) 295: 1928-1943, 2012.

13. Strupp M, Zwergal A, Feil K, Bremova T and Brandt T: Pharmacotherapy of vestibular and cerebellar disorders and downbeat nystagmus: Translational and back-translational research. Ann N Y Acad Sci 1343: 27-36, 2015.

14. Wegner I, van Benthem PP, Aarts MC, Bruintjes TD, Grolman W and van der Heijden GJ: Insufficient evidence for the effect of corticosteroid treatment on recovery of vestibular neuritis. Otolaryngol Head Neck Surg 147: 826-831, 2012.

15. Yamanaka T, Sasa M, Amano T, Miyahara $\mathrm{H}$ and Matsunaga $\mathrm{T}$ : Role of glucocorticoid in vestibular compensation in relation to activation of vestibular nucleus neurons. Acta Otolaryngol Suppl 519: 168-172, 1995. 
16. Zhi-Yuan LI: An animal model of experimental vestibular dysfunction by intratympanically injecting arsanilic acid in mice. J Pharm Pract 26: 427-429, 2008.

17. Livak KJ and Schmittgen TD: Analysis of relative gene expression data using real-time quantitative PCR and the 2(-Delta Delta C(T)) method. Methods 25: 402-408, 2001.

18. Bonny C, Borsello T and Zine A: Targeting the JNK pathway as a therapeutic protective strategy for nervous system diseases. Rev Neurosci 16: 57-67, 2005.

19. Felszeghy K, Banisadr G, Nyakas C and Haour F: Dexamethasone downregulates chemokine receptor CXCR4 and exerts neuroprotection against Hypoxia/ischemia-induced brain injury in neonatal rats. Neuroimmunomodulation 11: 404-413, 2004

20. Zhang Z, Zhang Z, Artelt M, Burnet M and Schluesener HJ: Dexamethasone attenuates early expression of three molecules associated with microglia/macrophages activation following rat traumatic brain injury. Acta Neuropathol 113: 675-682, 2007.

21. Du XD, Li H and Liao L: Experimental study on protective effects of dexamethasone on traumatic brain injury in rats. Sichuan Da Xue Xue Bao Yi Xue Ban (Chinese) 40: 486-489, 2009.

22. Zhang Z, Fauser U and Schluesener HJ: Early attenuation of lesional interleukin-16 up-regulation by dexamethasone and FTY720 in experimental traumatic brain injury. Neuropathol Appl Neurobiol 34: 330-339, 2008.
23. Breivik T, Thrane PS, Gjermo P and Opstad PK: Glucocorticoid receptor antagonist RU 486 treatment reduces periodontitis in Fischer 344 rats. J Periodontal Res 35: 285-290, 2000.

24. Ning Q, Yang H, Li E, Li C and Xu C: Effect of corticosterone on the activity and gene expression of ornithine decarboxylase in rat regenerating hepatocytes. Dong Wu Xue Bao 50: 778-783, 2004.

25. Rivera C, Li HCJ, Lahtinen H, Viitanen T, Nanobashvili A, Kokaia Z, Airaksinen MS, Voipio J, Kaila K and Saarma M: BDNF-induced TrkB activation down-regulates the $\mathrm{K}+-\mathrm{Cl}-$ cotransporter $\mathrm{KCC} 2$ and impairs neuronal $\mathrm{Cl}$ - extrusion. J Cell Biol 159: 747-752, 2002.

26. Mcbride CB, Mcphail LT and Steeves JD: Emerging therapeutic targets in caspase-dependent disease. Expert Ther Targets 3: 391-411, 1999.

27. Repici M and Borsello T: JNK pathway as therapeutic target to prevent degeneration in the central nervous system. Adv Exp Med Biol 588: 145-155, 2006.

28. Shuto M, Higuchi K, Sugiyama C, Yoneyama M, Kuramoto N, Nagashima R, Kawada K and Ogita K: Endogenous and exogenous glucocorticoids prevent trimethyltin from causing neuronal degeneration of the mouse brain in vivo: Involvement of oxidative stress pathways. J Pharmacol Sci 110: 424-436, 2009. 